\title{
ITERATIVE USE OF SIMULATION AND SCHEDULING METHODOLOGIES TO IMPROVE PRODUCTIVITY
}

\author{
Karthik Krishna Vasudevan \\ Ravi Lote \\ Edward J. Williams \\ Onur Ülgen \\ Production Modeling Corporation \\ 15726 Michigan Avenue \\ Dearborn, MI 48126 USA
}

\begin{abstract}
Experienced and wise industrial engineering educators and practitioners have long understood that industrial engineering is a coherent discipline encompassing techniques that work best synergistically, not a motley collection of specialized techniques each isolated in a separate chimney. As an example of the synergies which industrial engineering can bring to process improvement in a production environment, this case study presents the integrated use of process simulation, production scheduling, and detailed analysis of material-handling methods and their improvement. The study undertook the identification and improvement of production and scheduling policies to the benefit of a manufacturing process whose original throughput capacity fell significantly short of high and increasing demand.
\end{abstract}

\section{INTRODUCTION}

Inherent variability of manufacturing processes, spaghetti flow caused by looping process flows or multiple rework loops and its susceptibility to changes in the supply chain make manufacturing systems very complex. Drill collar manufacturing in particular is plagued by long cycle times at each process step, unwieldy part dimensions, bulk and fragility in addition to the above. In this paper, we apply Simulation and Scheduling methodologies to address the aforementioned complexities.

Historically, one of the first and traditionally most prominent practical uses of simulation has been its application to the manufacturing sector of the economy (Law and McComas 1999). This use of simulation is especially valuable when the manufacturing process must have integrated within it significant material-handling equipment and hence constraints (Krieg, Völker, and Geipel 1996), as often happens when the items of manufacture are heavy, bulky, and/or fragile. The synergy of analytical techniques for simulation and scheduling in combination is steadily becoming both more widely known and more powerful in manufacturing (Collier and Evans 2007).

In a manufacturing context, (Giribone, Mosca, and Queirolo 2003) applied simulation to development and validation of weekly production schedules. In a service industry context, (Zottolo, Williams, and Ülgen 2007) applied simulation to the scheduling of work by classification at various car-rental agency offices at diverse airports. (Otamendi 2007) describes algorithms and software to apply simulation to the scheduling of geographically dispersed maintenance activities. In the present context, due to the bulk and weight of the items being manufactured and hence moved among various operations, simulation additionally helped significantly improve material-handling resource allocation and leveling, and operations overall, as envisioned in the tutorial work of (Manivannan 1998). An practical example of simulation heavily devoted to material-handling concerns appears in (Kodeekha 2004).

Scheduling is widely used in the manufacturing environment as a tool to assign production tasks to resources while satisfying several system constraints. Modern scheduling algorithms use a combination of heuristic and brute-force approaches to compute the feasibility and effectiveness of many possible assignment patterns. A large number of researches have employed various methods such as mathematical programming, dispatching rules, expert systems, neural networks, genetic algorithms, and inductive learning to design scheduling systems for manufacturing applications (Jones and Rabelo 1998). Wysk et al. (1986) developed an integrated expert system/simulation scheduler called MPECS which used deterministic simulations to evaluate expert system schedules in a lab environment. Kutanoglu and Sabuncuoglu (2001) presented an experimental investigation of iterative simulation based scheduling in a job shop environment.

The key contribution of this work is in the real-word (large manufacturing system) application of the iterative 
use of simulation results as an input to scheduling and the schedules so generated as a simulation input parameter. The capability of scheduling tools to analyze thousands of alternatives quickly combined with the power of simulation to provide detailed statistical insights into specific scenarios allows these methods to effectively complement each other. This two layer approach is used for the optimal design of manufacturing system fed with robust schedules thus resulting in substantial productivity gains.

\section{OVERVIEW OF MANUFACTURING SYSTEM}

\subsection{Introduction}

Drill Collars are components of the drill string (drilling equipment) that are used to provide weight to the bit for drilling. Drill collars are thick-walled tubular pieces machined from solid bars of steel, usually plain carbon steel but sometimes of nonmagnetic nickel-copper alloy or other nonmagnetic premium alloys. The bars of steel are drilled from end to end to provide a passage to pumping drilling fluids through the collars. Widely used in the oil drilling industry, drill collars can measure 30 feet in length and weigh up to $10,000 \mathrm{lbs}$.

The facility under consideration manufactures drill collars of various weights, sizes, shapes and alloy compositions. The manufacturing process involves a series of material (alloy) removal operations. The input to the system are large "raw alloy bars" that need to be machined to specifications to create the collar. These raw alloy bars are sent to the facility from a supplier according to a schedule fixed by management based on customer orders. The raw alloy bars arrive in trucks and are moved into a factory floor space using material handling equipment.

The factory is organized into two shops. There exists some buffer space outside the building, to accommodate excess bars during peak truck hours. Each shop has bridge crane rails running along its walls with two cranes running on each (hence a total of four cranes). All the machines and storage spaces (buffers) inside the building are loaded and unloaded using these cranes.

\subsection{Process Flow}

The incoming bars are unloaded from the trucks using the bridge crane. The bridge crane loads these bars into the input buffer. The first processing step is the Peeling operation where the outside of the bar is scaled or peeled. Peeled bars are stored back into the input buffer. The bar is then gagged. The gagged bar is stored in a buffer and then tested using the ultrasonic test operation. The bars are then stored again in the input buffer. The bars that fail the ultrasonic test must re-visit the machine before shipping. The bars now are transported on the AGV [automated guided vehicle] three bars at a time across shops.
The bars are picked up from the AGV using the back crane in the second shop and stored in the incoming staging area. The next step in bar processing is the Cutting and Stamping. After Stamping they are moved into the CStage buffer. The $\mathrm{C}$ and $\mathrm{G}$ machines are used to drill through the length of the bar. Once a C-Machine is available, these bars are loaded on to the machine. The CMachines have relatively long operating times ranging from 2 to 10 hours. The stage buffer which feeds to the GMachines is immediately downstream of the C-Machines. The bars are tested after load and before processing at both the $\mathrm{C}$ and $\mathrm{G}$ machines. In case of failure at $\mathrm{C}$ Machines, they are sent to the Re-Gagging buffer from where they need to be transported to the first shop to get re-gagged. Bars that fail at the $\mathrm{G}$ machine need to be Honed for which they are sent to the Hone machine.

Once the bars are passed and worked on at the GMachine, they are loaded into the Pre-magnetic test buffer where they wait to undergo the Magnetic Test. Magnetic testing is carried out only during the night shift to prevent external magnetic interference. Bars that pass the Magnetic Test are stored in the Post testing buffer before they are sent across again into the first shop for polishing. The above process flow is represented as a flow diagram in Figure 1.

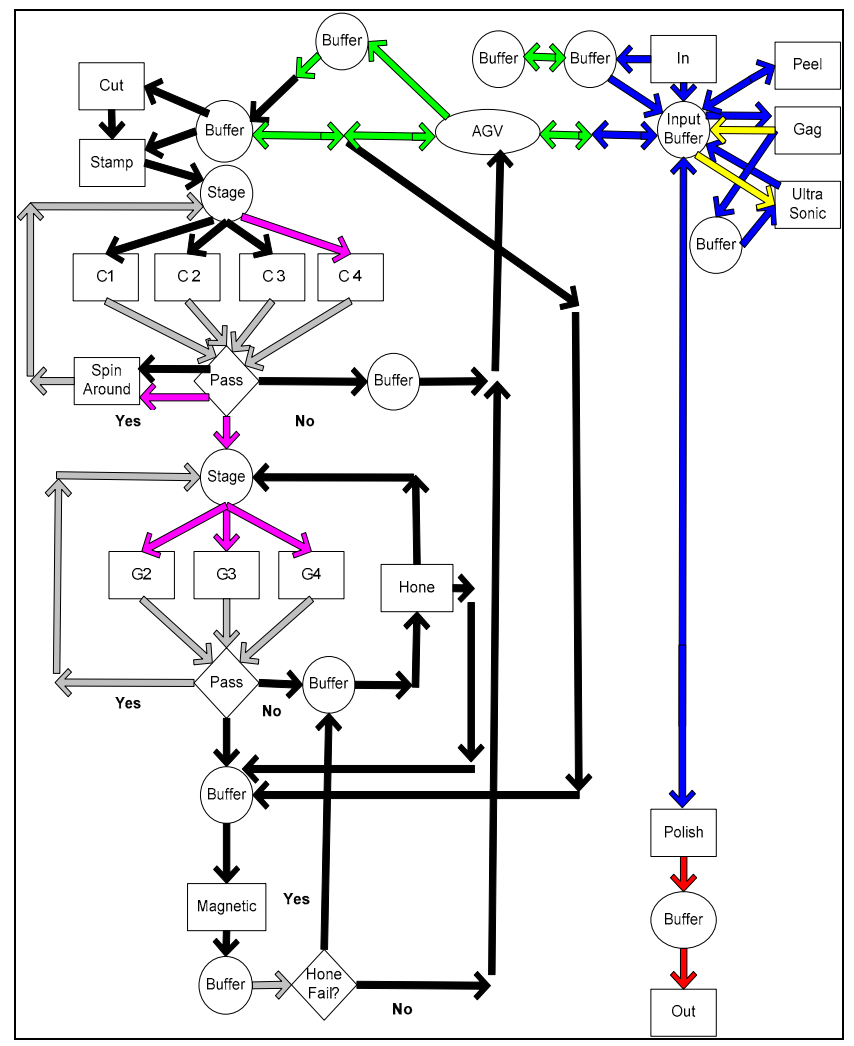

Figure 1: Process Flow 
In Figure 1 on the previous page, blue and red lines represent movements of Crane 1 and Crane 2 respectively in the "B" shop. Gray and magenta lines represent movements of Crane 1 and Crane 2 respectively in the "A" shop. Black lines represent transfers required to accomplish rework. Green lines represent movements of the AGV.

\subsection{Challenges}

The rise in the need for fossil fuels has lead to a spike in demand for drill collars. Hence, demand far outpaces production. The plant faces a number of challenges with respect to the throughput improvement efforts required to meet the ever increasing demand for drill collars. An effective, well tested, and high ROI - Throughput Improvement Roadmap was the need of the hour. Changing plant configurations and material handling systems is an expensive and arduous procedure owing to the size of equipment and parts. Long cycle times at each machine restrict the practical effectiveness of the Measure-Improve-AnalyzeControl cycle and the identification of the bottleneck by manual inspection of the line. The plant has no control over part sequence or priority changes. Schedules can be changed at the last minute by planners at regional planning centers. Inefficient sequencing results in excessive and costly changeovers that may last up to six hours on certain machines. The plant hence required not only a dynamic analysis of their process flow, but also an effective method to react in real-time to changes while meeting ambitious productivity targets.

\section{METHODOLOGY}

In order to effectively respond to the challenges described in Section 2.3, the consulting industrial engineers designed a two-layer methodology. Simulation was adopted as a tool to model the production flow, allowing us to test the robustness of possible throughput improvement efforts. Analysis of simulation results indicated the sensitivity of the throughput patterns to sequence of production assignments. This led to the use of the second layer consisting of scheduling methods to identify optimal sequences and to answer the "when to put which part on which machine?" question. Ineffective schedules were not only constraining system performance, but also caused repercussions down the line due to sudden non-availability of priority parts. Using a Scheduling tool also allows the plant to use both proactive and reactive methods to respond to sequence and priority changes while maintaining productivity levels. The schedule so generated is then used as an input into the simulation

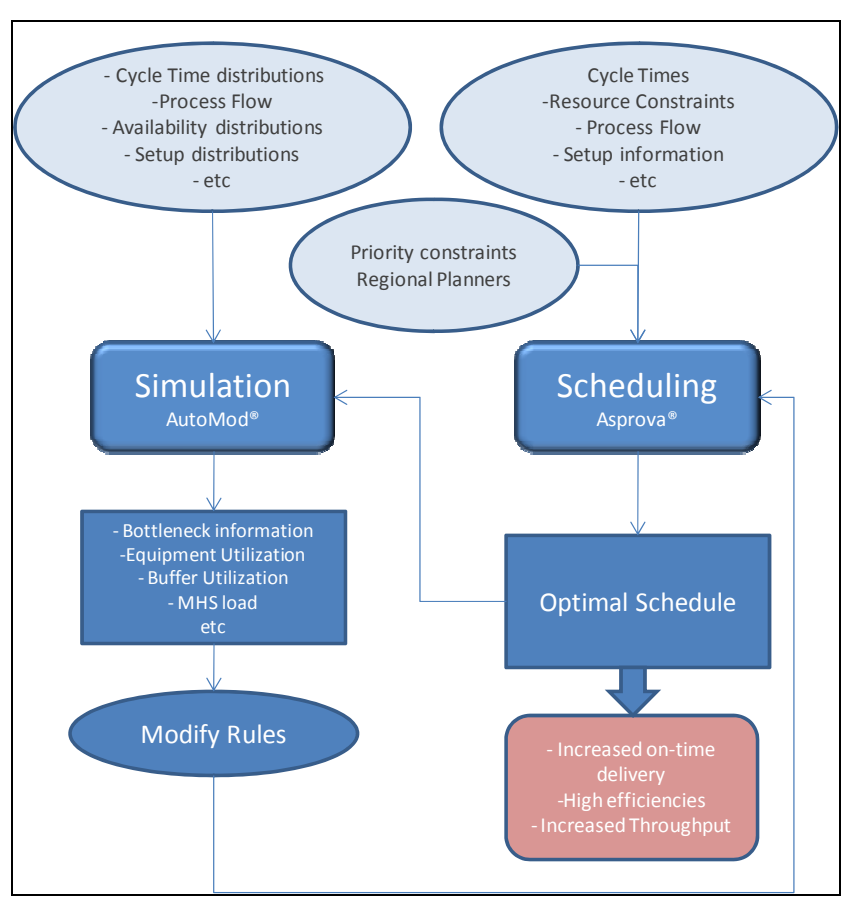

Figure 2: Methodology Block Diagram

The optimal schedule generated by Asprova ${ }^{\circledR}$ is used as an input into AutoMod ${ }^{\circledR}$ (Rohrer and $\mathrm{M}^{\mathrm{c}}$ Gregor 2002) to test the robustness to variability patterns in cycle times and availabilities. The effect of material handling systems (MHS), WIP levels etc. can be studied using the simulation. Optimal resource (including effect of MHS) allocations can then be fed back into Asprova ${ }^{\circledR}$ to generate better schedules. This iterative process is the backbone of the throughput improvement efforts in this project.

\section{DATA COLLECTION AND ANALYSIS}

\subsection{Input Data Collection}

Significant efforts were invested in collecting input data from the plant. The process was studied and the process flow was mapped based on information collected from plant experts. Historical plant databases were used to record various part sequences that were run during each day for a span of six months. Downtime data was also collected from the factory information system. Cycle times are a function of part weight. Hence a lookup table is used to reference each part type against a calculated processing time on each machine (based on part weight in lbs and metal removal rate $-\mathrm{lbs} / \mathrm{hr}$ ).

$$
\text { CycleTime }(\text { Bar i in Machine } j)=\frac{\text { Wieght }_{i}}{\text { ProcessingRate }_{j}}
$$


Setup times vary based on part diameters. Setups can range from one to six hours based on whether processing involves a change in the bar's internal diameter, external diameter, or both. The machines' availability also depends on their use for certain types of non-drill collar parts (these parts are not in the scope of the model). Hence machine availability is modeled as a fraction of a 10-day cycle (e.g. Machine A is available to process drill collars for 5 out of 10 days). Crane and AGV speeds were used to calculate travel times in the simulation model. Several data points for travel times were collected to verify these times. Load, unload, and buffer sorting times were also collected. All the above data, as Excel $\mathbb{R}$ input, is read into the simulation and scheduling models.

\subsection{Input Data Analysis}

The input data collected from the plant was fitted to various kinds of distributions for use in the simulation model. Based on observed patterns, all the cycle times were modeled as a uniform distribution with a $10 \%$ spread around the mean. MTBF and MTTR data were fitted to exponential distributions. The load, unload, and sorting times for the material handling systems were modeled as triangular distributions. Arithmetic means of the data were used in the deterministic scheduling model.

\section{MODELING, VERIFICATION \& VALIDATION}

This section deals with the steps and techniques involved in building both of the models (simulation and scheduling) built as a part of this project. The data interchange between these two models is also discussed.

\subsection{Simulation Model}

The process flow was converted into modeling logic in

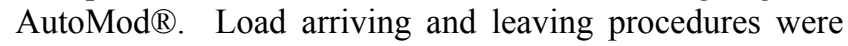
used to describe the flow of parts through various stages of the process. Resource constraints and processing times at each resource were used in these procedures. The states of these resources were tracked manually. Several state data (blocked, starved, off-shift etc) besides the default Auto$\operatorname{Mod}{ }^{\circledR}$ states (busy, idle, down) were recorded. Material handling system functions were used to model load and unload times and to control their motion. Counters were extensively used to prevent deadlock occurrences in the model. The model initialization function was used to read in data from specific sheets and cells in Excel®. Machine availabilities, processing rates, part dimensions, fixed sequences, buffer size details, etc. were read into the model from Excel ${ }^{\circledR}$.

The model was verified through repeated experiments and use of the debugger. Use of the three-dimensional animation ensured functionalities in the model. The simulation was run at high speeds for extended periods of time to ensure absence of model blockages. Output data from the verified model were recorded in Excel ${ }^{\circledR}$. Reports were formatted for easy readability and comprehensive analysis. Time series data recorded during model runs were used to validate the model against historical data obtained from the plant. Throughput histograms, availability charts, etc. were plotted and checked against plant data. Key issues identified during plant operation such as high crane utilization during certain shifts were also witnessed in the model. After consulting with plant SMEs the verified and validated simulation model was deemed fit for results collection and analysis.

\subsection{Scheduling Model}

The scheduling model is built in Asprova ${ }^{\circledR}$. The process flow is described through the Integrated Master Editor (IME) table, allowing definition of part-specific routings and resource dependencies. The Item table is used to describe the various kinds of drill collars (internal diameter, external diameter etc.). The Resource table is used to describe and group resources based on their functions. The Calendar table and the Spec Setup Tables are used to model shifts and changeover times based on item and resource information. Orders for specific parts are then placed using the Order table. Asprova ${ }^{\circledR}$ uses advanced scheduling algorithms to assign and peg operations (derived from exploding orders using the IME) to resources in the model. The combination of assignments - "the schedule" - is then evaluated based on resource allocation preference rules defined by the scheduler. Keeping in mind the goals of the study, the following five rules are employed in this model: (1) setup time minimization, (2) wait time minimization, (3) lateness minimization, (4) production time minimization, and (5) load leveling.

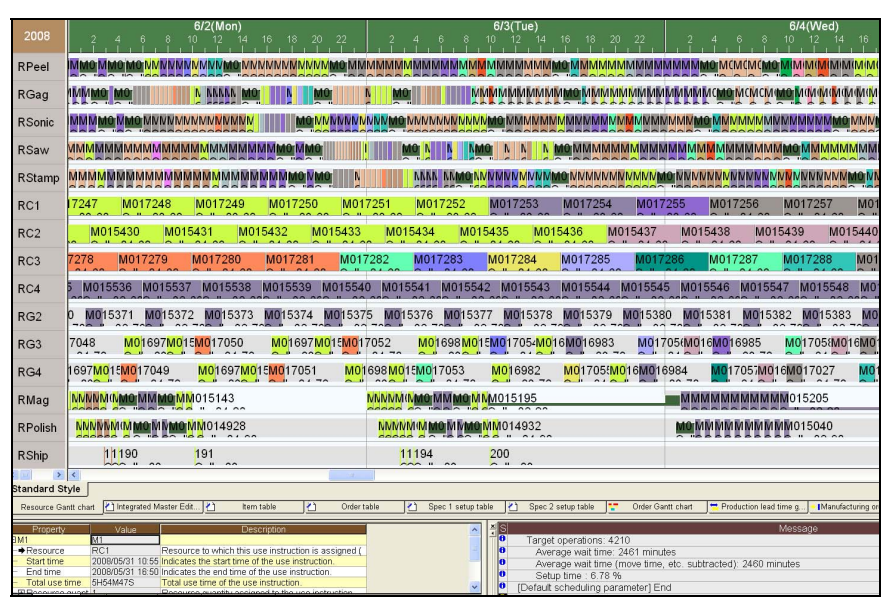

Figure 3: Resource Gantt Chart in Asprova ${ }^{\circledR}$ 


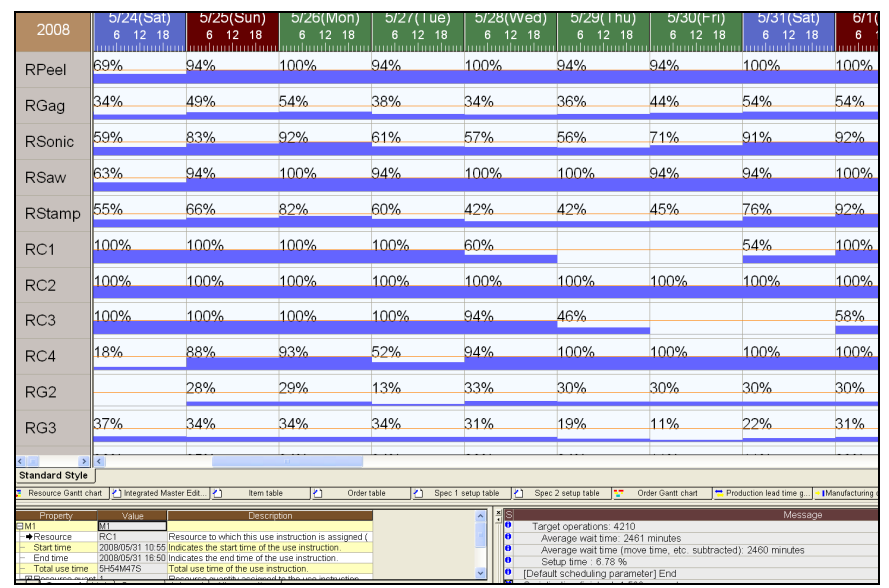

Figure 4: Resource Utilization Graph in Asprova ${ }^{\circledR}$

The best possible schedule based on the above rules is then shown on the resource Gantt chart (Figure 3). Resource utilization graphs (Figure 4) and lead time graphs allow the scheduler to foresee the effectiveness of the schedule. Sudden priority changes and special requests can be accommodated by re-organizing the operations on the resource Gantt chart. All other resources and operations are re-allocated and performance parameters are updated based on changes made. The generated schedule (array of operations) is exported into Excel ${ }^{\circledR}$ for easy import into AutoMod®. Production orders and dispatch lists are also generated using custom formatted reports.

Verification of the scheduling model involved errorproofing using graphical outputs. Lead times were verified to be reasonable and validated against plant data. The model was also validated using a historical schedule that was run for a month's worth of production. Resource utilizations and setup time percentages were compared against historical data available during the considered month.

\subsection{Iterative Use}

The verified and validated simulation model was run using base model settings. The time-in-state charts and other key performance indicators that were generated showed the sensitivity of the system to product mix and production sequence. Evaluation of past production schedules showed "muda" resulting from excessive setups. In order to validate the observation, common-sense changes were made to the schedule and the simulation model was re-run. A dramatic increase in throughput resulted.

Hence drawing from the results of the simulation, the Asprova ${ }^{\circledR}$ model was then built, verified, and validated. The schedule so generated had significantly lower setups even after several priority constraints were applied. The improved schedule was then used to fix the simulation's production sequence. The schedule was tested rigorously using uncertainties. The simulation showed a throughput increase and clear bottleneck areas, and exposed several weak resource priority and buffer size constraints. Such constraints were altered and this information was used to make changes in the scheduling model. The schedule was regenerated and the cycle was repeated. This iterative use of simulation and scheduling was repeated three times in this study to create/test the throughput improvement roadmap and make productivity improvement recommendations (discussed in Section 6). The continued iterative use of the models during daily production planning was also recommended.

\section{RESULTS AND RECOMMENDATIONS}

The simulation verified that the current system cannot meet the target throughput. Several improvements are suggested to achieve throughput to satisfy burgeoning demand. A three-stage bottleneck analysis was performed. During the base model run, the TIS chart showed that all the machines upstream of the magnetic test station has large blocked times. However the magnetic test and downstream machines had a significant starved time. Thus the system bottleneck was identified to be the post magnetic test buffer due to its inability to accumulate enough bars to feed the polish station (this station is available only 3 out of 10 days to process drill collars). It was suggested that these two operations be de-coupled by adding buffer capacity on the floor. This allows the efficient utilization of the polish station (when it is available). A $25 \%$ increase in throughput was observed in the simulation model as a result of the decoupling efforts.

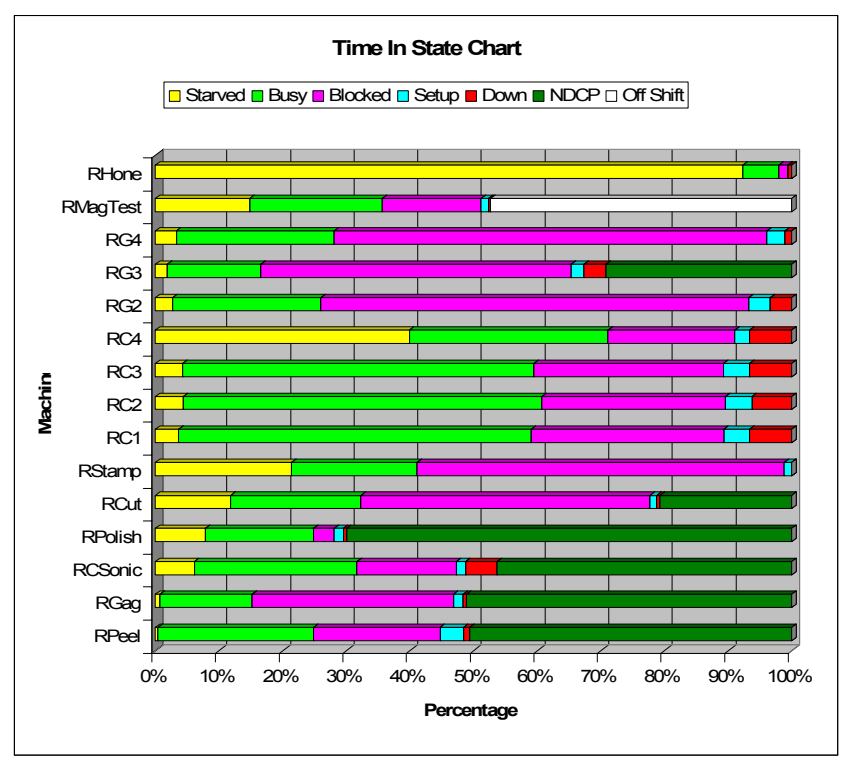

Figure 5: Base Model Time-In-State Chart

The simulation model was re-run with the de-coupling to uncover two parallel stage II bottlenecks. The availabil- 
ity of several upstream machines (peel, gag, ultrasonic) was found to be starving all the subsequent machines. The importance of optimizing schedules of non-drill collar parts was brought to the forefront. A $10 \%$ increase in availability yielded a $36 \%$ throughput increase over the base model. Also, alleviating the stress on the material handling system by adding a new crane in Shop 2 was seen to produce similar improvement effect on the throughput. However, combining both the above resulted in a $47 \%$ increase in simulation system throughput. The throughput improvement road map summarizing the recommendations is shown in Figure 6.

Table 1: Productivity Improvement Results

\begin{tabular}{|c|l|c|c|}
\hline Scenario & \multicolumn{1}{|c|}{ Action } & Bars/mo & \% \\
\hline Base & - & 237 & - \\
\hline I & Use "best" schedules & 266 & $12 \%$ \\
\hline II & De-couple Polish & 298 & $25 \%$ \\
\hline III & Better NDCP schedule & 324 & $36 \%$ \\
\hline IV & Add Shop 2 crane & 327 & $38 \%$ \\
\hline V & Both III \& IV & 347 & $47 \%$ \\
\hline
\end{tabular}

Using the schedules generated from Asprova ${ }^{\circledR}$ resulted in a $12 \%$ reduction in setup times when tested against a schedule used to run the same mix in the past. This translates to around 30 collars per month. Better load leveling patterns were also observed. On-time deliveries on last-minute orders improved considerably when tested in the simulation model.

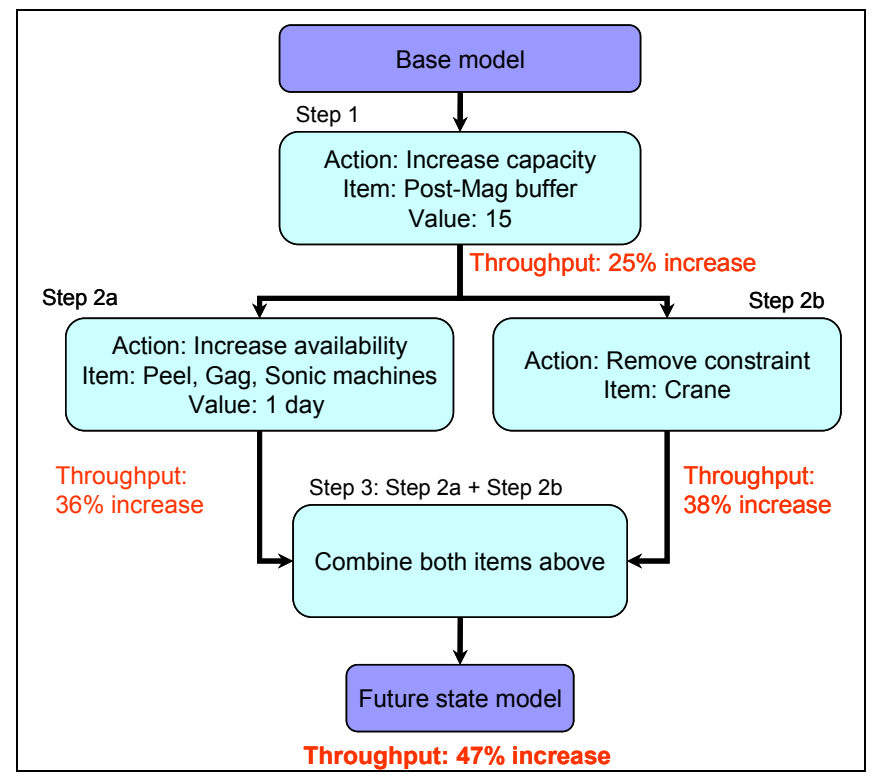

Figure 6: Throughput Improvement Roadmap

After discussing the incorporation of the above changes, it was seen that the non-availability of operators to run the slowest machine ( $\mathrm{C}$ machines) would constrain the system on the production floor. The prospect of reallocating operators from $\mathrm{G}$ machines (down stream of $\mathrm{C}$ machines) was recommended to increase availability of the $\mathrm{C}$ machines to increase throughput. Setups using the SMED (Single Minute-Exchange of Dies) approach to alleviate stress on the bottleneck were also recommended.

\section{CONCLUSION}

The iterative use of simulation and scheduling is presented as a powerful technique for making all-round productivity improvement recommendations. Recommendations were made to improve productivity by $47 \%$ resulting in an annual revenue increase of approximately $\$ 1,800,000$.

\section{ACKNOWLEDGMENTS}

The authors gratefully acknowledge the project leadership and guidance of Steve Beeler, Senior Project Manager, PMC.

\section{REFERENCES}

Collier, D. A., and J. R. Evans. 2007. Operations Management: Goods, Services and Value Chains. 2nd ed. Mason, Ohio: Thomson Higher Education.

Giribone, P., R. Mosca, and F. Queirolo. 2003. Validating the Production Weekly Plan by Scheduling Simulation. In Proceedings of the $15^{\text {th }}$ European Simulation Symposium, ed. Alexander Verbraeck and Vlatka Hlupic, 332-336.

Jones, A., and L. C. Rabelo, 1998. Survey of job shop scheduling techniques. Technical report, National Institute of Standards and Technology, Gaithersburg, MD.

Kodeekha, E. 2004. A New Method of FMS Scheduling Using Optimization and Simulation. In Proceedings of the $16^{\text {th }}$ European Simulation Symposium, ed. György Lipovszki and István Molnár, 29-34.

Krieg, M., M. Völker, and T. Geipel. 1996. Interaktive Kopplung zwischen Fertigungsleitstand und Materialflußsimulator. In Simulationstechnik 10. Symposium in Dresdsen, ed. Wilfried Krug, 61-64.

Kutanoglu, E., and I. Sabuncuoglu. 2001. Experimental Investigation of Iterative Simulation-Based Scheduling in a Dynamic and Stochastic Job Shop. Journal of Manufacturing Systems 20(4):264-279.

Law, A. M., and M. G. McComas. 1999. Simulation of Manufacturing Systems. In Proceedings of the 1999 Winter Simulation Conference, Volume 1, ed. Phillip A. Farrington, Harriet Black Nembhard, David T. Sturrock, and Gerald W. Evans, 56-59.

Manivannan, M. S. 1998. Simulation of Logistics and Transportation Systems. In Handbook of Simulation: 
Principles, Methodology, Advances, Applications, and Practice, ed. Jerry Banks, 571-604.. New York, New York: John Wiley \& Sons, Incorporated.

Otamendi, Javier. 2007. Simulation-Optimization in Geographic maintenance Scheduling. In Proceedings of the $21^{\text {st }}$ European Conference on Modelling and Simulation, ed. Ivan Zelinka, Zuzana Oplatková, and Alessandra Orsoni.

Rohrer, M. W., and I. W. $\mathrm{M}^{\mathrm{c}}$ Gregor. 2002. Simulating Reality Using AutoMod. In Proceedings of the 2002 Winter Simulation Conference, Volume 1, ed. Enver Yücesan, Chun-Hung Chen, Jane L. Snowdon, and John M. Charnes, 173-181.

Wysk, R. A., D. Wu, and R. Yang. 1986. A multi-pass expert control system (MPECS) for flexible manufacturing systems. NBS Special Publication 724:251-278.

Zottolo, M., E. J. Williams, and O. M. Ülgen. 2007. Simulation Implements Demand-Driven Workforce Scheduler for Service Industry. In Proceedings of the 2007 Winter Simulation Conference, ed. S. G. Henderson, B. Biller, M.-H. Hsieh, J. Shortle, J. D. Tew, and R. R. Barton, 219-225. CD ISBN: 1-4244-1306-0.

\section{AUTHOR BIOGRAPHIES}

KARTHIK KRISHNA VASUDEVAN is an Applications Engineer at Production Modeling Corporation (PMC), Dearborn, Michigan, USA. His technical skills span simulation, software, controls and scheduling domains with experience in AutoMod $\AA$, Witness ${ }^{\circledR}$, Simul8 $\AA$, Extend $\AA$, Emulate $3 \mathrm{D} \AA, \mathrm{VB}, \mathrm{C}++$ and Asprova ${ }^{\circledR}$. He received a Master's degree in Industrial Engineering from the University of Arizona, Tucson, USA and a Bachelor's degree in Electrical and Electronics Engineering from Sathyabama University, Chennai, India. He received the "Dean's award for Outstanding Graduate Student" and the "Award for Excellence" at the University of Arizona. His career and research interests lie in multi-paradigm simulation modeling and analysis and in the efficient integration of various IE techniques and software systems with computer simulation for creating better business strategies. He is a professional member of the IIE (Institute of Industrial Engineers), ASQ (American Society of Quality) and MSUG (Michigan Simulation User Group).

RAVI LOTE is a Consulting Project Manager at PMC. Over the last ten years, Ravi has built simulation models for dozens of customers in the U.S. and overseas. His functional areas of expertise include simulation modeling, process improvement and supply chain optimization. Ravi has a Bachelors' Degree in Mechanical Engineering from Shivaji University, India and a Masters' Degree in Industrial Engineering from the University of Massachusetts, Amherst. He is currently pursuing an M.B.A. from the University of Michigan, Ann Arbor. Ravi is a certified Six
Sigma Black Belt and a certified MODAPTS ${ }^{\circledR}$ professional for conducting Industrial Engineering time studies.

EDWARD J. WILLIAMS holds bachelor's and master's degrees in mathematics (Michigan State University, 1967; University of Wisconsin, 1968). From 1969 to 1971, he did statistical programming and analysis of biomedical data at Walter Reed Army Hospital, Washington, D.C. He joined Ford Motor Company in 1972, where he worked until retirement (December 2001) as a computer software analyst supporting statistical and simulation software. After retirement from Ford, he joined PMC, Dearborn, Michigan, as a senior simulation analyst. Also, since 1980, he has taught evening classes at the University of Michigan, including both undergraduate and graduate simulation classes using GPSS $/ \mathrm{H}^{\mathrm{TM}}$, SLAM II ${ }^{\mathrm{TM}}$, SIMAN ${ }^{\mathrm{TM}}$, ProModel ${ }^{\circledR}$, SIMUL8 $\AA$, or Arena $\AA$. He is a member of the Institute of Industrial Engineers [IIE], the Society for Computer Simulation International [SCS], and the Michigan Simulation Users' Group [MSUG]. He serves on the editorial board of the International Journal of Industrial Engineering - Applications and Practice. During the last several years, he has given invited plenary addresses on simulation and statistics at conferences or seminars in Monterrey, México; İstanbul, Turkey; Genova, Italy; Rīga, Latvia; Göteborg, Sweden; and Jyväskylä, Finland. He has served as Program Chair of the 2004, 2005, and 2006 Summer Computer Simulation Conferences, and also for the 2005 IIE Simulation Conference. In May 2008, he was the Track Coordinator for the simulation track at the annual Institute of Industrial Engineers conference. His university web page is: $<$ http://www-personal.umd.umich.edu/ williame> .

ONUR M. ÜLGEN is the president and founder of Production Modeling Corporation (PMC), a Dearborn, Michigan, based industrial engineering and software services company as well as a Professor of Industrial and Manufacturing Systems Engineering at the University of MichiganDearborn. He received his Ph.D. degree in Industrial Engineering from Texas Tech University in 1979. His present consulting and research interests include simulation and scheduling applications, applications of lean techniques in manufacturing and service industries, supply chain optimization, and product portfolio management. He has published or presented more that 100 papers in his consulting and research areas. Under his leadership PMC has grown to be the largest independent productivity services company in North America in the use of industrial and operations engineering tools in an integrated fashion. PMC has successfully completed more than 3000 productivity improvement projects for different size companies including General Motors, Ford, DaimlerChrysler, Sara Lee, Johnson Controls, and Whirlpool. The scientific and professional societies of which he is a member include Ameri- 
Vasudevan, Williams, Lote, and Ülgen

can Production and Inventory Control Society (APICS) and Institute of Industrial Engineers (IIE). He is also a founding member of the MSUG (Michigan Simulation User Group). 